\title{
Numerical Solution of Plateau's Problem by a Finite Element Method
}

\author{
By Masahiro Hinata, Masaaki Shimasaki and Takeshi Kiyono
}

\begin{abstract}
This paper concerns the application of a finite element method to the numerical solution of a nonrestricted form of the Plateau problem, as well as to a free boundary problem of Plateau type. The solutions obtained here are examined for several examples and are considered to be sufficiently accurate.

It is also observed that the hysteresis effect, which is a feature of a nonlinear problem, appears in this problem.
\end{abstract}

1. Introduction. Methods for the numerical solution of the Plateau problem have so far been examined by D. Greenspan [3], [4], using the combination technique of difference and variational methods, and by P. Concus [5], using a finite difference method.

These two methods can be applied only to the so-called restricted form of the Plateau problem described by Forsythe and Wasow [2, Section 18.9], that is, to the problems where the boundary condition is represented by a single-valued function. Thus, they cannot be applied to the problem where the boundary condition is represented by a multi-valued function, such as Courant's example described later.

This paper shows that such multi-valued boundary-value problems can be solved numerically by a finite element method. In this case, two solution methods, one for a free boundary problem and the other in a cyclindrical coordinate system, are presented.

2. Application of a Finite Element Method to the Plateau Problem. The Plateau problem involves finding a twice continuously differentiable function $u(x, y)$ in a region $D$ satisfying

$$
\left.\begin{array}{rl}
u(x, y) & =f(x, y), \quad \text { or } \\
\partial u(x, y) / \partial x & =g(x, y) \\
\partial u(x, y) / \partial y & =h(x, y)
\end{array}\right\} \text { on } \partial D
$$

and minimizing the surface area functional

$$
J u=\iint_{D}\left(1+u_{x}^{2}+u_{y}^{2}\right)^{1 / 2} d x d y,
$$

where $f(x, y), g(x, y)$ and $h(x, y)$ are single-valued functions [2, Section 18.9].

Received June 7, 1972.

AMS (MOS) subject classifications (1970). Primary 49D15; Secondary 49F10, 65P05.

Key words and phrases. A boundary-value problem, a finite element method, a free boundary problem, cylindrical coordinate system, R. Courant's example, the hysteresis effect of Plateau's problem. 


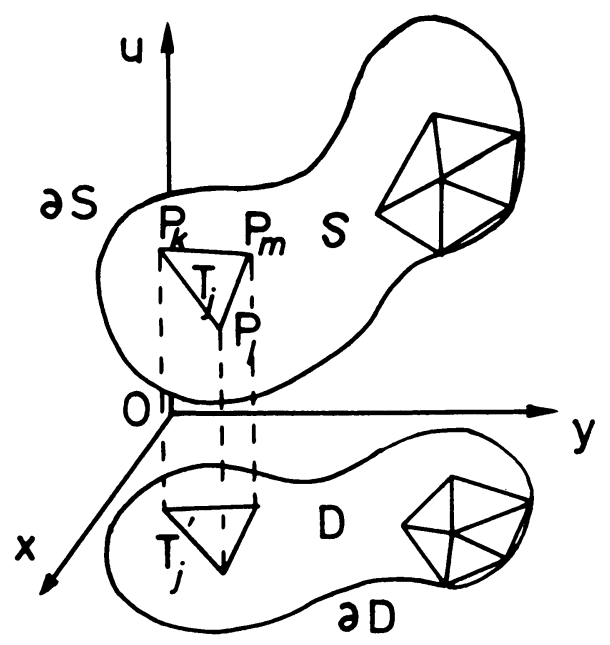

Figure 1. A coordinate system

A finite element method is applied to the Plateau problem in the following manner: Consider a linear basis function $u_{j}(x, y)$ written in the form

$$
u_{i}(x, y)=A_{i} x+B_{i} y+C_{i} .
$$

The coefficients $A_{i}, B_{i}$ and $C_{i}$ in Eq. (2.3) can be determined by three points in the $x-y-u$ space. Let $D$ be approximated by the union of disjoint triangles $T_{i}{ }^{\prime}(j=$ $1, \cdots, Q)$. Let the three vertices of $T_{i}{ }^{\prime}$ in the $x-y$ plane be $P_{k}{ }^{\prime}\left(x_{k}, y_{k}, 0\right), P_{l}{ }^{\prime}\left(x_{l}, y_{l}, 0\right)$, and $P_{m}^{\prime}\left(x_{m}, y_{m}, 0\right)$ and let the three vertices of a triangle $T_{i}$ in the $x-y-u$ space corresponding to $T_{i}^{\prime}$ be $P_{k}\left(x_{k}, y_{k}, u_{k}\right), P_{l}\left(x_{l}, y_{l}, u_{l}\right)$ and $P_{m}\left(x_{m}, y_{m}, u_{m}\right)$, respectively. Then $T_{i}$ is represented in the form

$$
u_{j}(x, y)=\left(\left|\begin{array}{lll}
u_{k} & y_{k} & 1 \\
u_{l} & y_{l} & 1 \\
u_{m} & y_{m} & 1
\end{array}\right| x+\left|\begin{array}{ccc}
x_{k} & u_{k} & 1 \\
x_{l} & u_{l} & 1 \\
x_{m} & u_{m} & 1
\end{array}\right| y+\left|\begin{array}{lll}
x_{k} & y_{k} & u_{k} \\
x_{l} & y_{l} & u_{l} \\
x_{m} & y_{m} & u_{m}
\end{array}\right|\right)\left|\begin{array}{lll}
x_{k} & y_{k} & 1 \\
x_{l} & y_{l} & 1 \\
x_{m} & y_{m} & 1
\end{array}\right| .
$$

The area of the triangle $T_{i}{ }^{\prime}$ is given by

$$
\iint_{T_{i}{ }^{\prime}} d x d y=\frac{1}{2} \text { abs }\left|\begin{array}{ccc}
x_{k} & y_{k} & 1 \\
x_{l} & y_{l} & 1 \\
x_{m} & y_{m} & 1
\end{array}\right|
$$

and the area of the polyhedral surface consisting of the $T_{i}$ is given by

$$
\begin{aligned}
J u=\tilde{J} u & =\sum_{j=1}^{Q} \iint_{T_{j^{\prime}}}\left(1+u_{i x}^{2}+u_{i v}^{2}\right)^{1 / 2} d x d y \\
& =\sum_{i=1}^{Q}\left(1+A_{i}^{2}+B_{i}^{2}\right)^{1 / 2} \iint_{T_{j^{\prime}}} d x d y \\
& =\sum_{i=1}^{Q} \frac{1}{2}\left(\left|\begin{array}{lll}
x_{k} & y_{k} & 1 \\
x_{l} & y_{l} & 1 \\
x_{m} & y_{m} & 1
\end{array}\right|^{2}+\left|\begin{array}{lll}
u_{k} & y_{k} & 1 \\
u_{l} & y_{l} & 1 \\
u_{m} & y_{m} & 1
\end{array}\right|^{2}+\left.\left|\begin{array}{lll}
x_{k} & u_{k} & 1 \\
x_{l} & u_{l} & 1 \\
x_{m} & u_{m} & 1
\end{array}\right|^{2}\right|^{1 / 2}\right.
\end{aligned}
$$






FIGURE 2. Schematic view of $f_{i}\left(z_{1}, z_{2}, \cdots, z_{p}\right)$

The problem of finding a twice continuously differentiable function $u(x, y)$, minimizing the continuous functional $J u$, can thus be approximated by that of finding a piecewise linear function

$$
\tilde{u}(x, y)=\sum_{j=1}^{0} u_{i}(x, y) \Phi_{i}(x, y)
$$

where

$$
\begin{array}{ll}
\Phi_{i}(x, y)=1 & \text { in } T_{i}^{\prime}, \\
\Phi_{i}(x, y)=0 & \text { out of } T_{i}^{\prime},
\end{array}
$$

minimizing the functional $J u$.

The following fact should be noted. In the finite element method, it is not necessary to fix the $T_{i}{ }^{\prime}$, or the division of $D$; it is only necessary to minimize the functional $J u$, treating the $P_{i}$ 's as unknown points in the $(x, y, u)$-space. This flexibility is useful in solving various problems, such as free boundary problems. If the variational point $P_{i}\left(x_{i}, y_{i}, u_{i}\right)$ is selected in the direction $\left(\alpha_{i}, \beta_{i}, \gamma_{i}\right)$ from the point $\left(x_{i}{ }^{0}, y_{i}{ }^{0}, u_{i}{ }^{0}\right)$, that is

$$
x_{i}=\alpha_{i} z_{i}+x_{i}^{0}, \quad y_{i}=\beta_{i} z_{i}+y_{i}^{0}, \quad u_{i}=\gamma_{i} z_{i}+u_{i}^{0},
$$

where $\alpha_{i}{ }^{2}+{\beta_{i}}^{2}+{\gamma_{i}}^{2}=1$, then the necessary condition for minimizing $J u$ is given by

$$
\partial \tilde{J} u / \partial z_{i} \equiv f_{i}=0 \quad(i=1, \cdots, p) .
$$

A numerical analyst can choose the direction of variation in a manner best suited to his problem.

Equations (2.12) are nonlinear algebraic equations, which may be solved iteratively by the generalized Newton method, in which the $(n+1)$ th approximation $z_{i}{ }^{(n+1)}$ to $z_{i}$ is determined by

$$
\begin{array}{r}
z_{i}^{(n+1)}=z_{i}^{(n)}-\omega \frac{f_{i}\left(z_{1}^{(n+1)}, \cdots, z_{i-1}^{(n+1)}, z_{i}^{(n)}, z_{i+1}^{(n)}, \cdots, z_{p}^{(n)}\right)}{\partial f_{i}\left(z_{1}^{(n+1)}, \cdots, z_{i-1}^{(n+1)}, z_{i}^{(n)}, z_{i+1}^{(n)}, \cdots, z_{p}^{(n)}\right) / \partial z_{i}}, \\
\quad(i=1, \cdots, p) ;
\end{array}
$$






FIGURE 3. Contour with a multi-valued boundary condition

here $\omega$ is a relaxation parameter which when sufficiently small results in convergence.

We now consider $f_{i}$ and $\partial f_{i} / \partial z_{i}$ in Eq. (2.13). A simple computation gives the following result:

$$
\begin{gathered}
\left|f_{i}\right|<M_{1}, \\
M_{2}>\left(\partial f_{i} / \partial z_{i}\right)>0,
\end{gathered}
$$

where $M_{1}$ and $M_{2}$ are constants.

An outline of $f_{i}$ as a function of $z_{i}$ is shown in Fig. 2. This figure shows that when $\partial f_{i} / \partial z_{i}$ is extremely small, the generalized Newton method diverges.

3. A Free Boundary Problem. Minimal surface problems with multi-valued boundary conditions cannot be solved numerically by the usual finite difference method [3]-[5]. Certain types of such problems, however, can be reduced to free boundary problems with single-valued boundary conditions. We will now examine such free boundary problems using the finite element method of the previous section. Consider the contour shown in Fig. 3. If $\theta>\pi / 2$, the contour cannot be projected in a single-valued fashion and a boundary-value function is not single-valued. To avoid the adverse condition of multi-valuedness, the surface was cut with the plane $x=0$, taking advantage of the symmetry of the solution (see Fig. 4). Treating only the space $x \geqq 0$, the surface under consideration becomes a single-valued function of $u$ and $y$, while the cross section of the surface and the $u-y$ plane becomes an unknown boundary curve (the free boundary $F$ in Fig. 4).

As boundary conditions, we have

$$
\begin{gathered}
x=r \cdot \sin (\pi-\theta) \quad \text { on } y=0,0 \leqq u \leqq L, \\
(y-r \cdot \cos (\pi-\theta))^{2}+x^{2}=r^{2}, \quad x \geqq 0, y \geqq 0 \text { on } u=0 \text { or } L,
\end{gathered}
$$




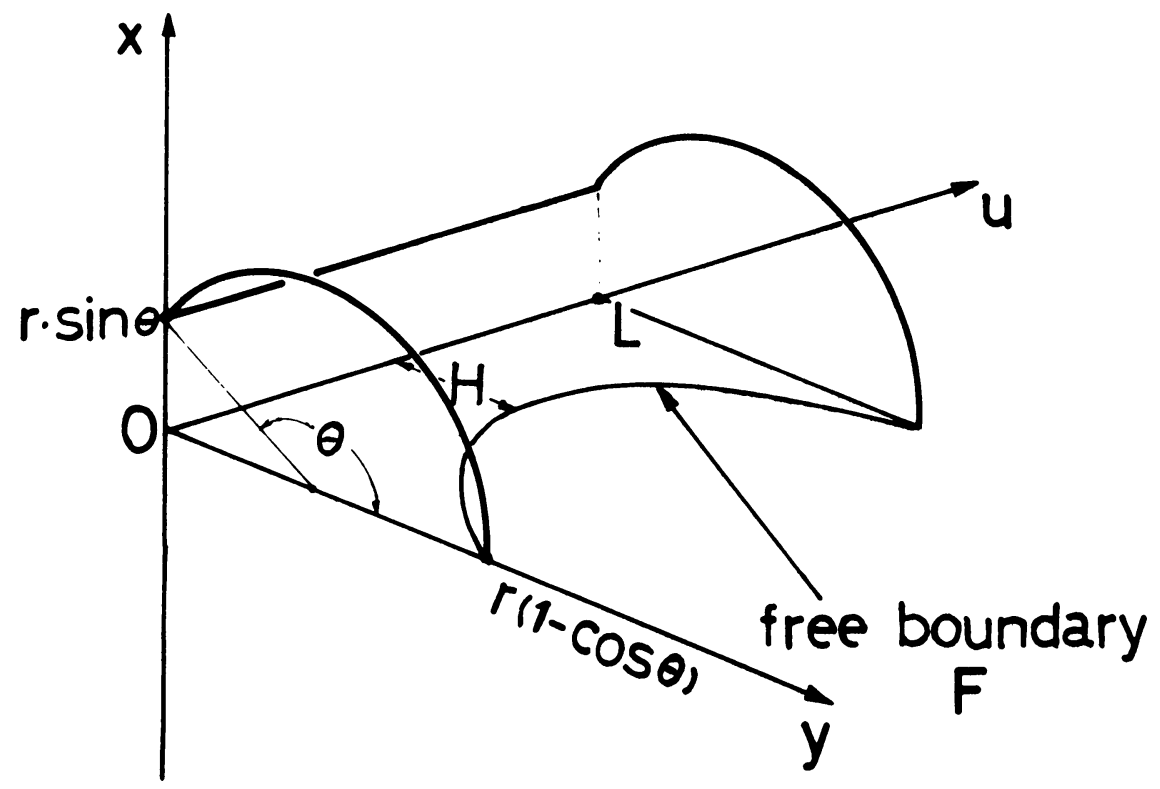

FIGURE 4. A free boundary scheme

and, on the free boundary, treating the unknown surface as a function of $x$ and $u$, we have

$$
\partial y / \partial x=0 \text { on } x=0, \quad 0 \leqq u \leqq L .
$$

Using the symmetry with respect to $u=L / 2$, the boundary conditions are eventually given by

$$
\begin{gathered}
x=r \cdot \sin \theta \text { on } y=0 \text { and } 0 \leqq u \leqq L / 2, \\
x^{2}+(y+r \cdot \cos \theta)^{2}=r^{2} \quad \text { on } u=0, x \geqq 0, y \geqq 0, \\
\partial y / \partial u=0 \text { on } x=0,0 \leqq u \leqq L / 2, \\
\partial x / \partial u=0 \text { on } u=L / 2,0 \leqq y \leqq H,
\end{gathered}
$$

where the unknown height $H$ from the plane $y=0$, i.e., the $x$ - $u$ plane, is determined by solving the above boundary problem.

On the free boundary $F$, the unknown variables are $y_{M, i}(j=1, \cdots, N)$, while in the region $D$, the unknown variables are $x_{j}(j=1, \cdots, p-N)$. Then the $y$ component of the point on the curve $F$ must be largest in $y$-components of points in $D$ which have the same $u$-component, and in the present program, $y_{i, i}$ is given by

$$
y_{i, i}=y_{M, i} \cdot y_{i, 0} / y_{M, 0}
$$

(see Fig. 5). Thus, the division of the region varies during the generalized Newton iterations and this flexibility is the key to the successful investigation of the free boundary problem.

We now describe some numerical examples. Here

$$
u_{i}=j \Delta u, \quad \Delta u=L /(2 N), \quad j=0,1, \cdots, N,
$$

and 


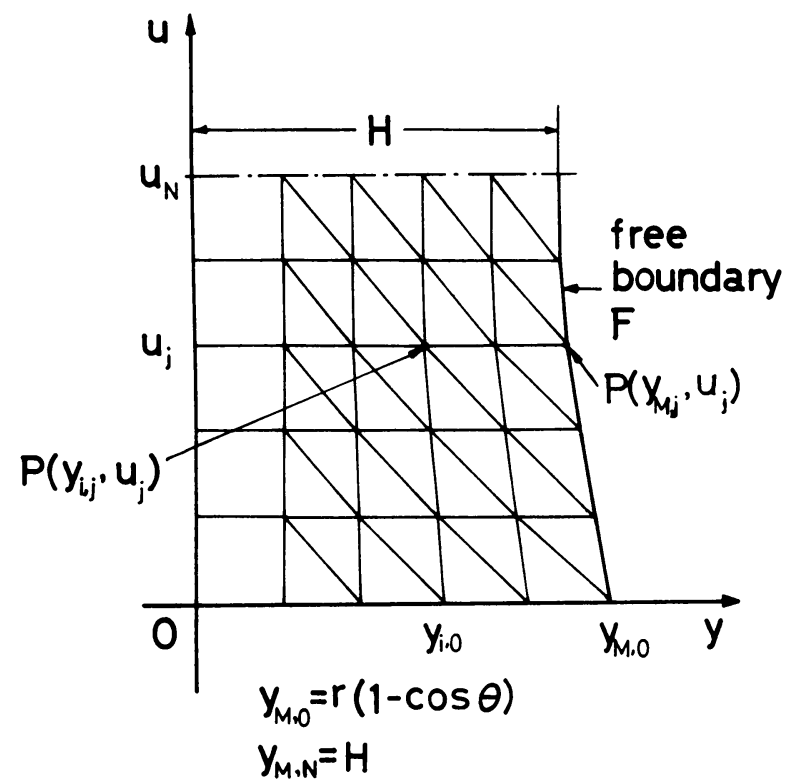

FIGURE 5. Division of the region for the free boundary problem



FigURE 6. Two directions of projection for the case $\theta=\pi / 2$

$$
y_{i, 0}=r\{1-\cos (i \Delta \theta)\}, \quad \Delta \theta=\theta / M, i=0,1, \cdots, M .
$$

Example 1. $\theta=\pi / 2, r=1.0, L=1.0$. This boundary-value problem was first solved numerically in direction $A$ (see Fig. 6) using Greenspan's method for which a theoretical analysis is given in [6]. The problem was then solved in direction $B$ with the present free boundary program. For comparison, numerical results given by these two methods are seen in Table 1, where the initial approximations are both 
TABLE 1. Comparison of two methods

\begin{tabular}{|c|c|c|}
\hline & $A$ & $B$ \\
\hline$H$ & 0.8552107 & 0.8551592 \\
\hline Area $(1 / 4)$ & 0.7571279 & 0.7571000 \\
\hline Relaxation parameter & $1.2^{*}$ & $1.2^{*}$ \\
\hline Iterations to convergence & 144 & 221 \\
\hline Number of arc divisions: $M(\Delta \theta)$ & $15\left(6^{\circ}\right)$ & $15\left(6^{\circ}\right)$ \\
\hline Number of $L / 2$ divisions: $N$ & 7 & 7 \\
\hline
\end{tabular}

* In this table, the initial relaxation parameter is 1.2 , and $\delta$, the termination parameter for iterations is $10^{-7}$. When $\max _{1 \leq i \leq p}\left|z_{i}^{(n+1)}-z_{i}^{(n)}\right|<\delta$, the iteration process is stopped.

cylindrical, namely

$$
x^{2}+y^{2}=1, \quad y \geqq 0,0 \leqq u \leqq L,
$$

Example 2. Catenoid. The second example is that of the catenoid. The soap film with this boundary condition is shown in Fig. 7. This problem can also be solved as a free boundary problem with boundary conditions (3.2)-(3.4) and the Neumann condition

$$
\partial x / \partial y=0 \text { on } y=0 \text { and } 0 \leqq u \leqq L / 2
$$

in place of (3.1); here $\theta=\pi / 2$.

$L$ and $a$ in Fig. 7 are connected by the relation

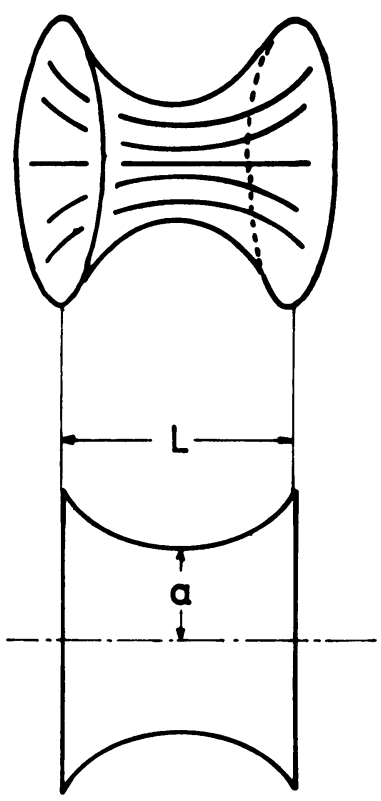

Figure 7. Catenoid 
TABLE 2. Comparison of two values of a

\begin{tabular}{ccc}
\hline & \multicolumn{2}{c}{$a$} \\
\cline { 2 - 3 }$L / 2$ & Value from Eq. (3.7) & $\begin{array}{c}\text { Value from free } \\
\text { boundary program }\end{array}$ \\
\hline 0.3 & 0.9523568 & 0.9520186 \\
\hline 0.4 & 0.9107381 & 0.9100505 \\
\hline 0.5 & 0.8483438 & 0.8470727 \\
\hline 0.6 & 0.7450713 & 0.7423604 \\
\hline 0.63 & 0.6937394 & 0.6894363 \\
\hline 0.66 & 0.5941309 & 0.5739461 \\
\hline 0.67 & no value & no value
\end{tabular}

$$
a \cdot \cosh (L a / 2)=1
$$

and $L$ must necessarily satisfy the relation

$$
L / 2 \leqq \lambda_{0} / \cosh \lambda_{0}
$$

where $\lambda_{0}$ is the root of the equation

$$
\lambda=\operatorname{coth} \lambda \text {. }
$$

It is seen that $\lambda_{0}$ is nearly equal to 1.2 , so that $\lambda_{0} / \cosh \lambda_{0}$ is nearly equal to 0.66 . For comparison, two computed values of $a$ are given in Table 2. One value was obtained from Eq. (3.7) and the other was computed as $H$ using the present free boundary program $\left(\Delta u=0.1, \Delta \theta=10^{\circ}\right)$. In this case again, the results of the present computer programs are close to the actual values.

Example 3. An example of $R$. Courant. We now apply the finite element method to the interesting contour shown in Figs. 8, 9, and Fig. 3, for $\theta>\pi / 2$. The soap film problem may have two different solutions, depending on the length of the line $L$ and the angle of $\operatorname{arc} \theta(\theta>\pi / 2)$ (see Figs. 8, 9). Let $\theta=5 \pi / 6, r=1.0$ and $L$ vary in Fig. 3. This boundary may sometimes be spanned by two different soap films



Figure 8. A solution of the soap film problem

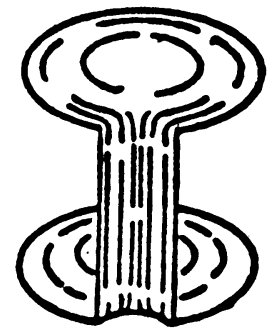

FIGURE 9. Another solution of the soap film problem 


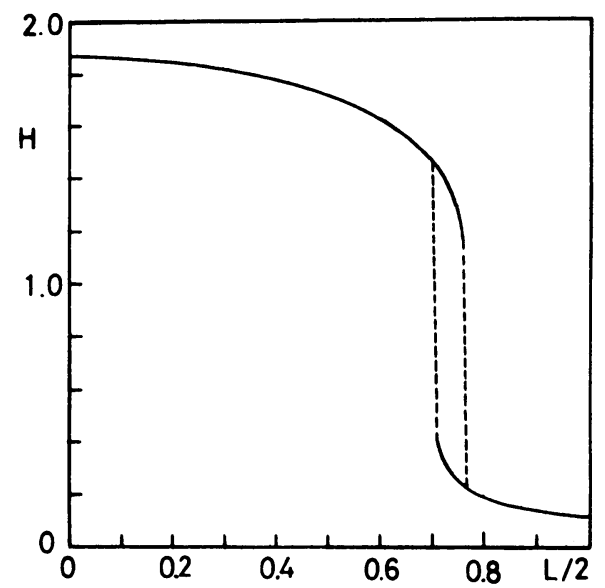

FIGURE 10. Relation between $L$ and $H$

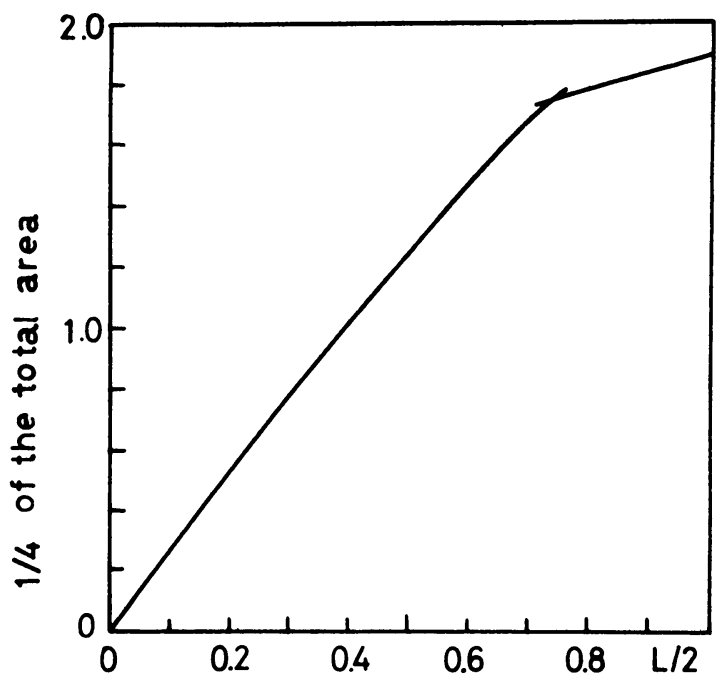

FIGURE 11. Relation between $L$ and $1 / 4$ of the total area

which we will now compute with the present free boundary program. The relation between $L$ and $H$, obtained by numerical computation, is shown in Fig. 10 and the relation between $L$ and $1 / 4$ of the total area in Fig. 11. Figures 10 and 11 show the hysteresis effect, which is a feature of a nonlinear problem.

We define the $n$th norm $N^{(n)}$ of solutions, the $n$th refinement $R^{(n)}$ and the $n$th eigenvalue $\eta^{(n)}$, by

$$
\begin{gathered}
N^{(n)}=\left(\sum_{i=1}^{p}\left(z_{i}^{(n)}\right)^{2}\right)^{1 / 2}, \\
R^{(n)}=\operatorname{abs}\left(N^{(n)}-N^{(n-1)}\right)
\end{gathered}
$$

and

$$
\eta^{(n)}=R^{(n)} / R^{(n-1)},
$$



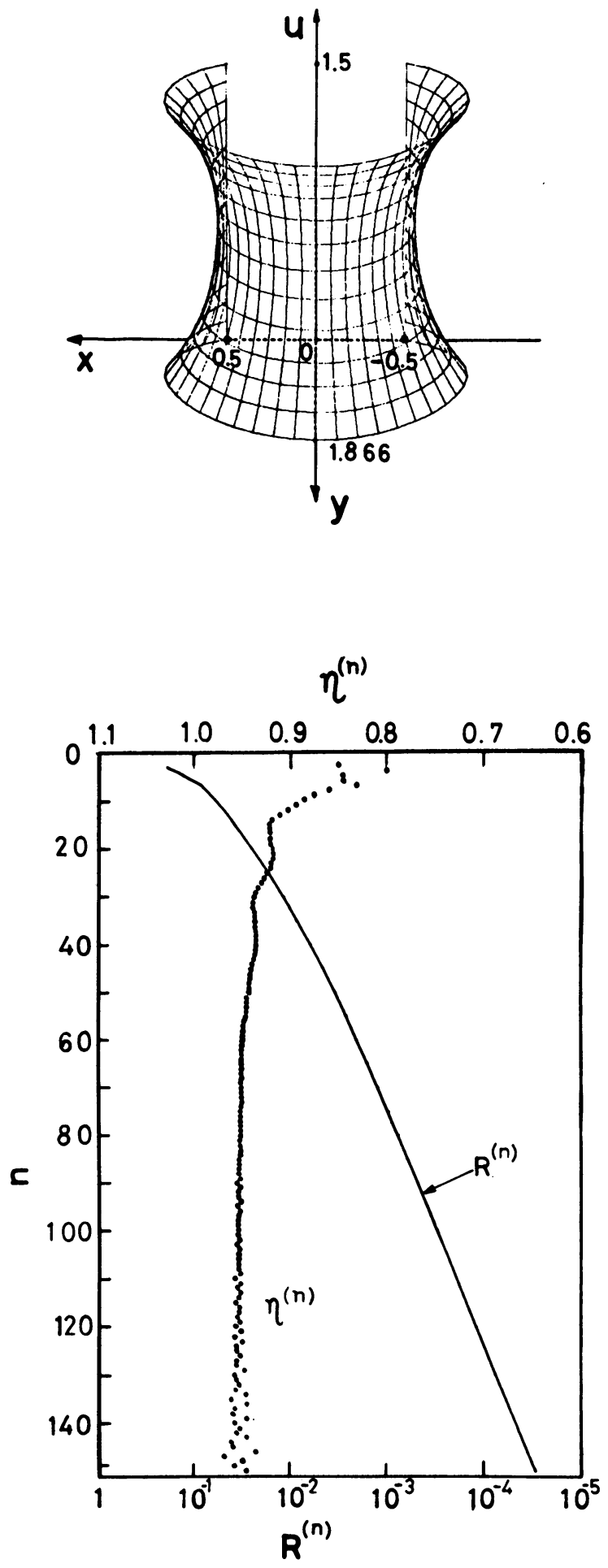

FIGURE 12. A solution for $L=1.5$ obtained by starting from an initial approximation of the cylinder $x^{2}+(y+\cos (5 \pi / 6))^{2}=1$ 

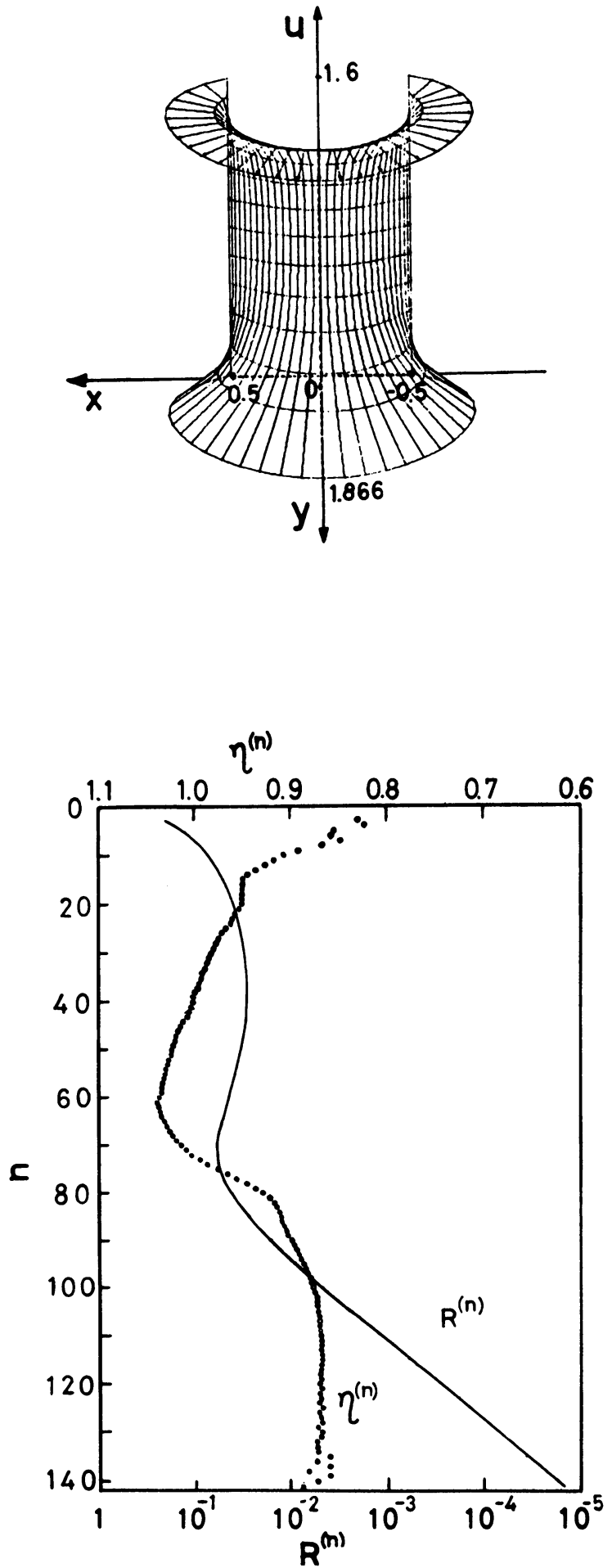

FIGURE 13. A solution for $L=1.6$ obtained by starting from an initial approximation of the cylinder $x^{2}+(y+\cos (5 \pi / 6))^{2}=1$ 

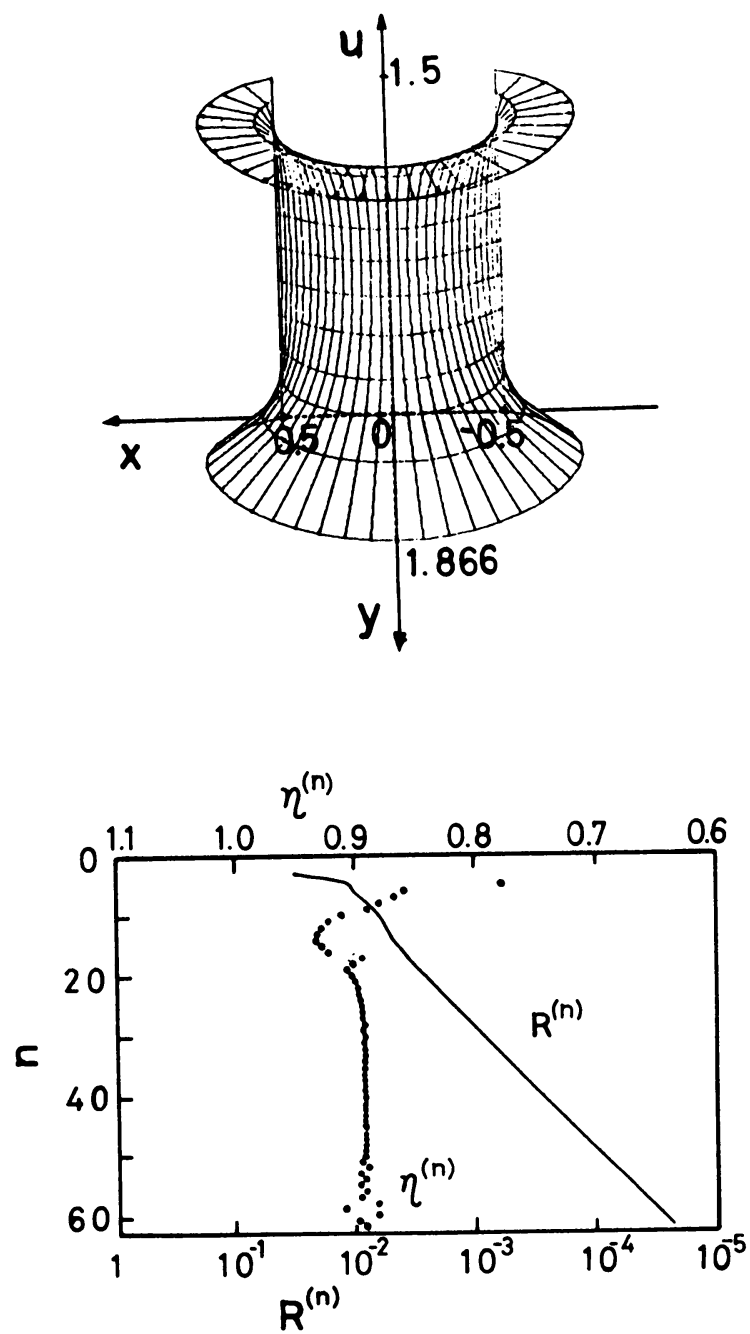

FIGURE 14. Another solution for $L=1.5$. This solution can be obtained by using the solution for $L=1.6$ shown in Fig. 16 as a starting approximation for this problem

respectively, where $p$ is the number of all unknown variables $z_{i}$. We show the starting approximation, the final approximate solution (converged) and $R^{(n)}, \eta^{(n)}$ in each iteration for two different solutions for $L=1.5$ in Figs. 12 and 14, respectively, where $\delta=10^{-5}$. The solution for $L=1.5$, shown in Fig. 14, was obtained starting from the final solution for $L=1.6$ shown in Fig. 13.

4. Cylindrical Coordinate System. We next consider the foregoing problem in cylindrical coordinates.

To avoid the boundary condition function being two-valued, this boundary is cut with the plane $u=u_{i}\left(0 \leqq u_{i} \leqq L\right)$ (see Fig. 15) and a cross section is shown in Fig. 16. 


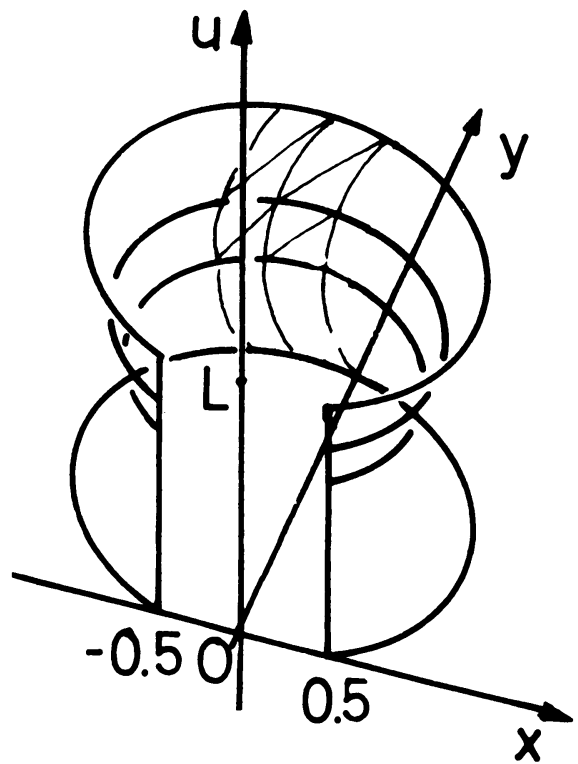

FIGURE 15. Cylindrical coordinate system for the example of $R$. Courant

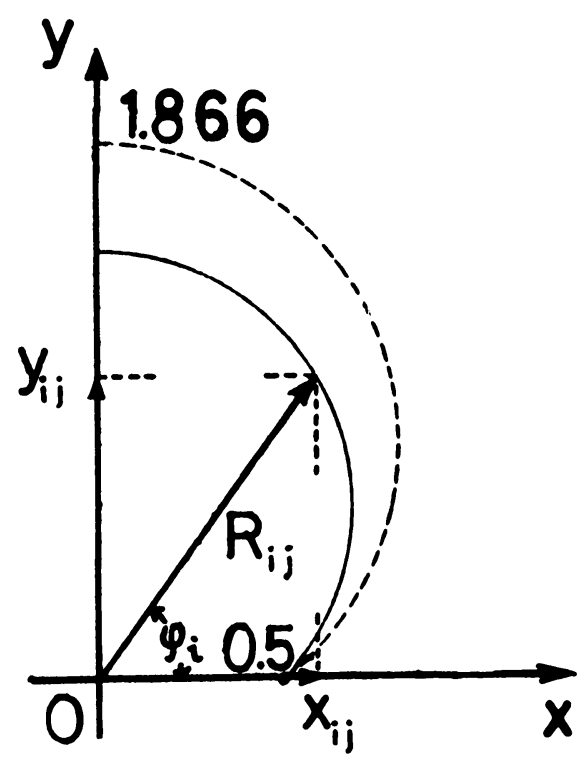

FIGURE 16. The cross section at $u=u_{j}$

The unknown variable $z_{i}$ in this case is $R_{i, i}$, as shown in Fig. 16, and it is connected with $x_{i, i}$ and $y_{i, j}$ by

$$
x_{i, j}=R_{i, j} \cos \varphi_{i}, \quad y_{i, i}=R_{i, j} \sin \varphi_{i} .
$$

Hence, 


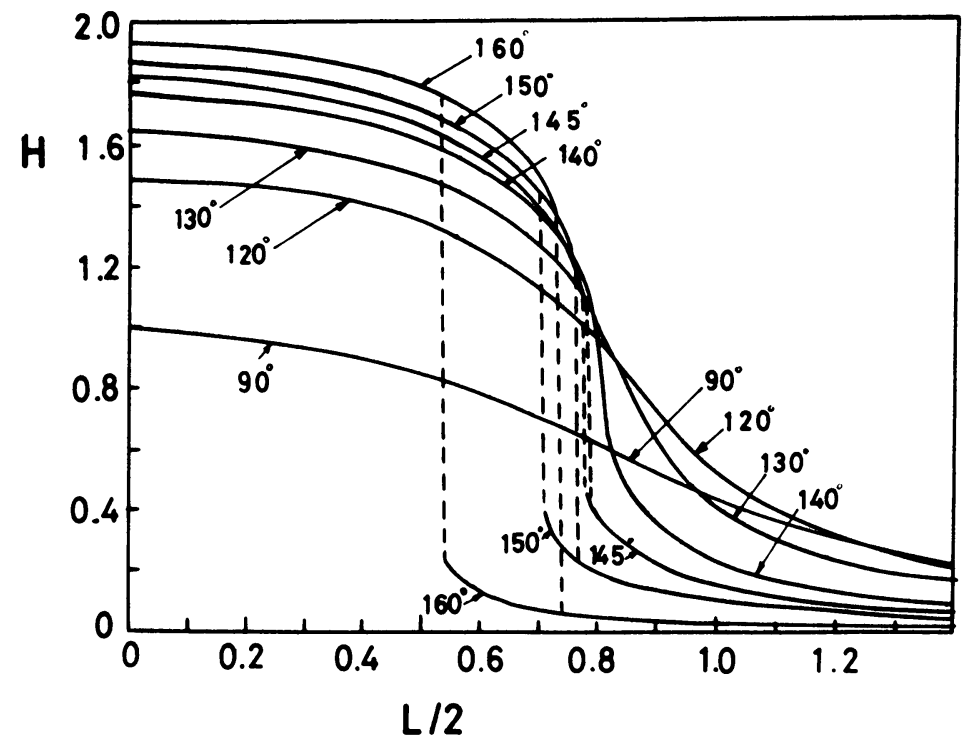

FIGURE 17. Relation between $L$ and $H$

$$
\begin{aligned}
\frac{\partial \tilde{J}_{u}}{\partial R_{i, j}} & =\frac{\partial J_{u}}{\partial x_{i, j}} \frac{d x_{i, j}}{d R_{i, j}}+\frac{\partial J_{u}}{\partial y_{i, j}} \frac{d y_{i, j}}{d R_{i, j}} \\
& =\frac{\partial \tilde{J}_{u}}{\partial x_{i, j}} \cos \varphi_{i}+\frac{\partial J_{u}}{\partial y_{i, j}} \sin \varphi_{i}=0 .
\end{aligned}
$$

For the comparison of a free boundary program and cylindrical coordinate system program $\left(u_{i}=L j / 12, \varphi_{i}=(\pi / 2) i / 15\right)$, numerical results of height $H$ and $\frac{1}{4}$ of the total area are given in Table 3, where $r=1.0, \theta=5 \pi / 6$ and $L$ varies.

TABLE 3. Comparison of two methods

C: cylindrical coordinate system

F: free boundary program

\begin{tabular}{cccc}
\hline$L / 2$ & & $H$ & $\frac{1}{4}$ of the total area \\
\hline \hline \multirow{2}{*}{0.7} & $\mathrm{C}$ & 1.447441 & 1.678319 \\
\cline { 2 - 4 } & $\mathrm{F}$ & 1.448845 & 1.678424 \\
\hline 0.75 & $\mathrm{C}$ & 1.256070 & 1.766360 \\
\cline { 2 - 4 } over & $\mathrm{F}$ & 1.260619 & 1.766551 \\
\hline 0.75 & $\mathrm{C}$ & 0.254281 & 1.754237 \\
under & $\mathrm{F}$ & 0.262425 & 1.756422 \\
\hline \multirow{2}{*}{0.8} & $\mathrm{C}$ & 0.2141318 & 1.782874 \\
\hline & $\mathrm{F}$ & 0.197830 & 1.785275 \\
\hline
\end{tabular}






FIGURE 18. The region where two different solutions exist

As shown in Table 3, the two values of $\frac{1}{4}$ of the total area by the two methods are in mutual agreement within $0.2 \%$ relative error and the values of $H$ within $3.5 \%$ for $L / 2 \leqq 0.75$ and $8.3 \%$ for $L / 2=0.8$. This fact would imply that the solution to Courant's problem presented here has sufficient accuracy.

5. Result of a More General From of R. Courant's Problem. Next, consider a more general form of Courant's problem. Here, $\theta$ is also taken to be a variable as well as $L . r=1.0$ was chosen.

Taking $\theta$ as a parameter, the relations between length $L$ and height $H$ are given in Fig. 17.

The region where the boundary has two solutions is given in Fig. 18.

6. Summary. It is shown that finite element methods are applicable to the Plateau problem. The variational direction suited to the problem can be selected in the finite element method and even the free boundary problem, which has been difficult to solve numerically, can be solved by the finite element method.

Even though the error analysis of the method presented here is yet to be fully examined, the examples considered give an insight into the accuracy of the computer program presented here. It is considered that the method has sufficient accuracy from a practical point of view.

All the computations were carried out using the FACOM 230-60 computer system at the Data Processing Center, Kyoto University.

Acknowledgement. One of the authors, M. Hinata, would like to thank Professor Shin Hitotsumatsu at the Research Institute for Mathematical Sciences, Kyoto University, for his helpful discussions. 
The authors would like to express their gratitude to the referee for his valuable comments.

Central Research Laboratories

Nippon Electric Company Ltd.

Kawasaki, Japan

Department of Information Science

Kyoto University

Kyoto, Japan

Department of Information Science

Kyoto University

Kyoto, Japan

1. R. Courant, Dirichlet's Principle, Conformal Mapping, and Minimal Surfaces, Interscience, New York, 1950. MR 12, 90.

2. G. E. FORSYTHE \& W. R. WASOW, Finite-Difference Methods for Partial Differential Equations, Appl. Math. Series, Wiley, New York, 1960. MR 23 \#B3156.

3. D. GREensPan, "On approximating extremals of functions. I: The method and examples for boundary value problems," ICC Bull., v. 4, 1965, pp. 99-120. MR 32 \#8526.

4. D. GReEnspan, "On approximating extremals of functions, Part II: Theory and generalization related to boundary value problems for nonlinear differential equations," Internat. J. Engineering Sci., v. 5, 1967, pp. 571-588. MR 36 \#3508.

5. P. Concus, "Numerical solution of the minimal surface equation," Math. Comp., v. 21,1967 , pp. $340-350$. MR 37 \#468.

6. R. STEPLEMANN, "Finite dimensional analogues of variational problems in the plane," SIAM J. Numer. Anal., v. 18, 1971, pp. 11-23. 